\title{
Cluster or QSO? Identifying 1RXSJ114720.0-125253
}

\author{
A. R. Zenn and H. Ebeling
}

Institute for Astronomy, University of Hawaii, 2680 Woodlawn Drive, Honolulu, HI 96822, USA

e-mail: anthonyz@hawaii.edu; ebeling@ifa.hawaii.edu

Received 22 July 2010 / Accepted 23 August 2010

\section{ABSTRACT}

\begin{abstract}
We present results from our analysis of a short Chandra observation of the unidentified X-ray source 1RXSJ114720.0-125253 detected in the ROSAT All-Sky Survey. Our 10 ks Chandra observation established unambiguously that the bulk of the X-ray emission $(85 \%)$ originates from the 6dF QSO g1147207-125310 $(z=0.496)$, although diffuse emission from a suspected alternative optical counterpart, the galaxy cluster LCDCS S031 $(z=0.580)$, is also detected. We measure X-ray luminosities of $(8.0 \pm 0.8) \times 10^{44} \mathrm{erg} \mathrm{s}^{-1}$ and $(2.7 \pm 0.4) \times 10^{44}$ for the QSO and the cluster, respectively $(0.3-8 \mathrm{keV})$. Both objects are thus found to be typical representatives of their respective population at the quoted redshifts. Thanks to over 1200 net photons having been detected from the QSO, we were able to characterize its spectrum further, finding a photon index of $1.4 \pm 0.1$ and no sign of significant self absorption. For the cluster, photon statistics (250 net photons) were too poor to constrain the intra-cluster gas temperature to better than (6.7 \pm 4.4$) \mathrm{keV}$, consistent with the estimate of $3.8 \mathrm{keV}$ from the $L_{\mathrm{X}}-k T$ relation. The cluster emission was also found to be significantly elongated and poorly described by a single $\beta$ model, suggesting an unevolved system or a recent merger event, a hypothesis supported also by the presence of a diffuse radio source about $200 \mathrm{kpc}$ from the cluster core. Prompted by this rare chance alignment of two X-ray luminous extragalactic sources, we discuss the probabilities of QSOs or clusters dominating the X-ray emission from RASS sources with ambiguous optical counterparts and apply the resulting probabilistic argument to the case of H1821+643.
\end{abstract}

Key words. X-rays: galaxies: clusters - quasars: individual: 1RXSJ114720.0-125253

\section{Introduction}

Galaxy clusters are fascinating targets for astrophysical study, allowing astronomers to probe a variety of topics, as diverse as the nature of dark matter or galaxy evolution as a function of environment. Clusters are the largest gravitationally bound objects in the Universe, arising from overdensities in the primordial density field to form objects of $10^{14-15}$ solar masses at the present epoch. The most massive clusters are extremely rare objects, and thus highly sensitive cosmological probes (Henry 2000; Borgani et al. 2001; Allen et al. 2003; Pierpaoli et al. 2003; Mantz et al. 2008).

Massive clusters can be readily identified by their X-ray characteristics, because the hot $\left(10^{7-8} \mathrm{~K}\right)$, diffuse intracluster gas residing in clusters emits primarily in the X-ray regime. By contrast, a cluster that appears optically rich and presumably massive as indicated by a high velocity dispersion of the cluster galaxies may actually be a poor unevolved system (or in fact not even gravitationally bound) whose apparent richness is inflated by projection effects and galaxy infall along the line of sight (Hicks et al. 2008; Horesh et al. 2010). Hence, X-ray surveys are uniquely suited for the compilation of cluster samples.

The MAssive Cluster Survey (MACS, Ebeling et al. 2001; Ebeling et al. 2007, 2010) was an X-ray cluster survey launched in 1998 with the explicit goal of finding the distant counterparts of the most massive clusters known in the local Universe. Based on X-ray detections from the ROSAT All-Sky Survey (RASS), MACS has vastly increased the number of very X-ray luminous clusters known, as illustrated in Fig. 1 which shows the luminosity-redshift distribution of clusters from various X-ray cluster surveys. Highlighted in this figure is the putative cluster MACSJ1147.3-1252 at $z=0.58$, the subject of this paper.

Throughout we used a $\Lambda$ CDM cosmology $\left(\Omega_{\mathrm{M}}=0.3, \Omega_{\Lambda}=\right.$ 0.7 ) and adopt $H_{0}=70 \mathrm{~km} \mathrm{~s}^{-1} \mathrm{Mpc}^{-1}$.

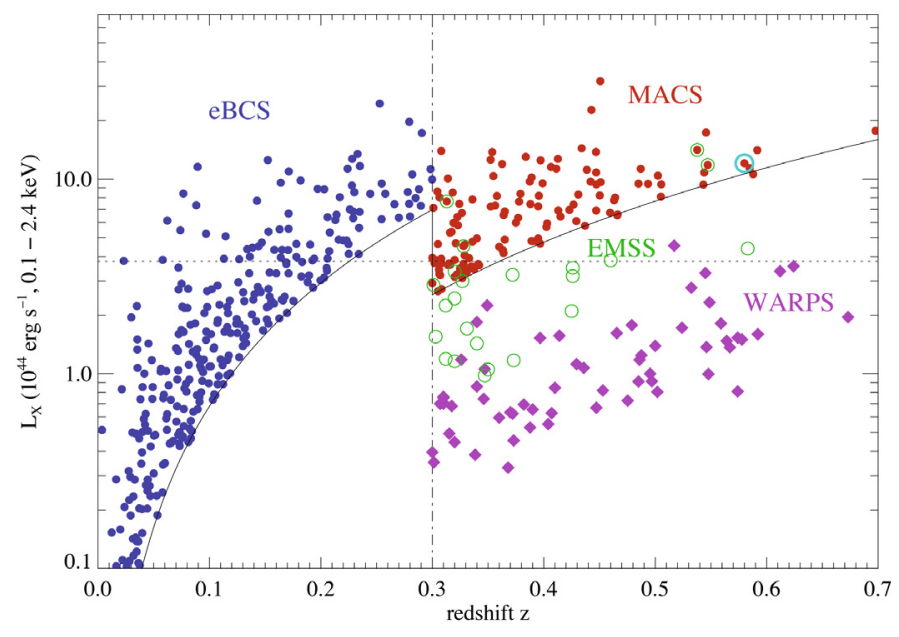

Fig. 1. Luminosity-redshift distribution of the clusters in the eBCS, EMSS, WARPS, and MACS samples. 1RXSJ114720.0-125253 is highlighted by a cyan circle, and note the relatively high X-ray luminosity and redshift value compared to other clusters in these samples. A $\Lambda \mathrm{CDM}$ cosmology and $h_{0}=0.7$ have been assumed.

\section{1RXSJ114720.0-125253}

1RXSJ114720.0-125253 is listed in the official RASS BrightSource Catalogue (BSC, Voges 1999) as an essentially pointlike source (at the angular resolution of the RASS) with 22 net photons detected in $326 \mathrm{~s}$, corresponding to an unabsorbed flux of $(1.16 \pm 0.32) \times 10^{-12} \mathrm{erg} \mathrm{s}^{-1} \mathrm{~cm}^{-2}(0.1-2.4 \mathrm{keV})$. The source's spectral hardness ratio ${ }^{1}$ of 0.05 only just exceeds the

\footnotetext{
${ }^{1}$ We use HR1, defined as $(b-a) /(b+a)$, where $a$ and $b$ are the count rates in the $0.11-0.41$ and the $0.52-0.2 \mathrm{keV}$ range.
} 
g1147207-125310 2004/06/09 z_helio $=0.49660 \mathrm{z}=0.49674$ qual $=4$

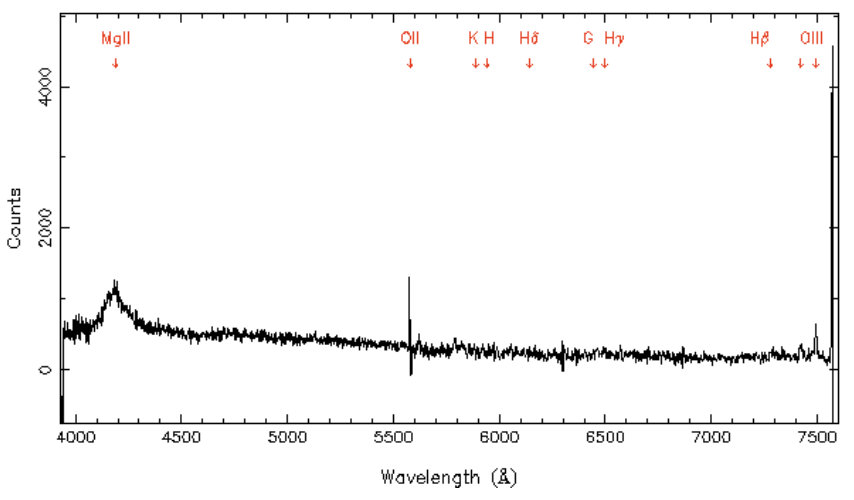

Fig. 2. Optical spectrum of the 6dF source g1147207-125310, identified as a QSO at $z=0.496$ (Jones et al. 2009). This object is less than 20 arcsec away from 1RXSJ114720.0-125253 and thus a highly plausible optical counterpart.

limit of -0.09 set for a cluster candidate at an $n_{\mathrm{H}}$ value of $2.92 \times 10^{20} \mathrm{~cm}^{-2}$ during the compilation of the MACS X-ray database (Ebeling et al. 2001). Owing to its low flux (the flux limit of MACS is $1 \times 10^{-12} \mathrm{erg} \mathrm{s}^{-1} \mathrm{~cm}^{-2}$ ) and relative spectral softness, 1RXSJ114720.0-125253 was not initially classified as a highly probable cluster and its identification thus only pursued toward the end of the MACS compilation phase.

1RXSJ114720.0-125253 was, however, flagged as a highpriority candidate in 2008, when optical follow-up observations with the University of Hawaii 2.2 m telescope revealed the presence of a potentially distant cluster. A literature search at this location found LCDCS S031 (Gonzales et al. 2001), a galaxy cluster with a spectroscopic redshift of $z=0.580$, less than an arcmin off the RASS X-ray source and coincident with the cluster visible in our UH2.2 $\mathrm{m}$ image. Also present in the literature, however, is a second plausible optical counterpart, about 20 arcsec away from 1RXSJ114720.0-125253, namely the GALEX source 2743230981068555158, spectroscopically identified as a QSO at $z=0.497$ by the $6 \mathrm{dF}$ survey (6dF id: $g 1147207-125310$, Fig. 2, Jones et al. 2009). Fig. 3 shows a UH2. 2 m colour image of a $3.5 \times 3.5 \mathrm{arcmin}^{2}$ area around LCDCS S031, illustrating the location of both potential counterparts with respect to 1RXSJ114720.0-125253.

If the bulk of the X-ray emission originates from LCDCS S031, i.e., a cluster at $z=0.580$, the system would be one of the most extreme clusters detected by MACS. Its location in the $L_{\mathrm{X}}-z$ plane, shown in Fig. 1), would make it the fourth most distant MACS cluster and, at $L_{X}=1.2 \times 10^{45} \mathrm{erg} \mathrm{s}^{-1}$, one of the 20 most X-ray luminous clusters known. Although the presence of a known QSO within the RASS error circle makes this scenario rather unlikely (based on the average space densities of either type of counterpart the probability of a cluster ID is in the percent range ${ }^{2}$ ), the rarity of extremely massive clusters at $z>0.5$ motivated further investigation of this source.

In order to determine if the bulk of X-ray emission originates from the cluster (defying the odds) or from the nearby QSO, we obtained an X-ray snapshot observation with the ACIS-I detector aboard the Chandra X-ray Observatory.

\section{Observations}

A $10 \mathrm{ks}$ observation of 1RXSJ114720.0-125253 was performed on February 19, 2009 using Chandra's ACIS-I detector

\footnotetext{
${ }^{2}$ See Sect. 7 for a detailed discussion of the underlying calculation.
}

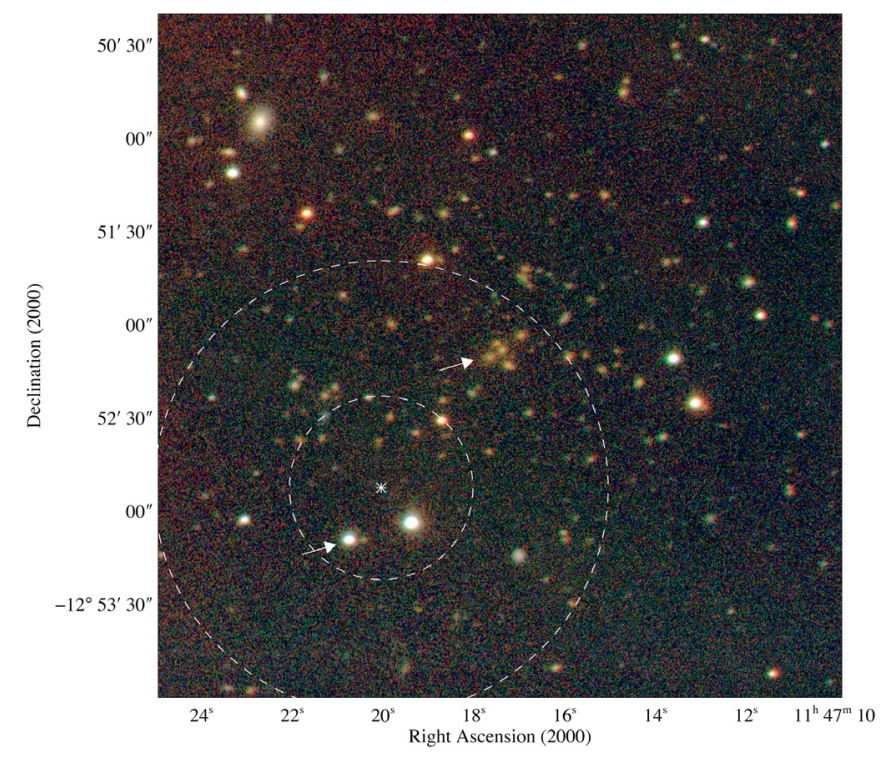

Fig. 3. VRI colour image of MACSJ1147.3-1252 obtained with the UH2. $2 \mathrm{~m}$ telescope in variable conditions in February 2008. The dashed lines mark the 1 and $2 \sigma$ error circles (derived using the entire MACS cluster sample) around the nominal RASS X-ray position for this 22photon detection. Arrows point to the two competing optical counterparts of this X-ray source: The 6dF quasar g1147207-125310 ( $z=$ $0.497)$ and the galaxy cluster LCDCS S031 $(z=0.580)$. (This figure is available in color in electronic form.)

(obsid 10414). The target was placed about 2.5 arcmin off the standard aimpoint (and thus almost 3 arcmin off axis) to avoid flux being lost to the chip gaps of the ACIS-I detector, while still maintaining good (sub-arcsec) angular resolution ${ }^{3}$ across the cluster core and the region around the possibly X-ray loud QSO. VFAINT mode was used in order to maximize the efficiency of particle-event rejection in the post-observation processing.

A visual inspection of the Chandra data obtained shows immediately that the vast majority of the X-ray emission originates from the QSO rather than the galaxy cluster (Fig. 4). Although we can thus already conclude that MACS1147.3-1252 is in fact not an extremely X-ray luminous cluster worthy of inclusion in the MACS sample, spectral and spatial analyses of the Chandra data in hand were performed to characterize both X-ray sources to the greatest possible extent.

\section{Analysis}

We used the CIAO software package (version 3.3) to reprocess the ACIS-I data and all other analysis tasks described in the following. Our inspection of the light curve of the event count rate in regions well away from obvious sources within the ACIS-I field of view found no significant flaring during the entire observation, leading to a good time interval of $9913 \mathrm{~s}$. We limit our analysis to the $0.3-8 \mathrm{keV}$ range, where Chandra has significant effective area and is least affected by cosmic rays or calibration uncertainties.

\subsection{Spatial modelling}

The response of ACIS-I is position and energy dependent. To correct for these effects, which are particularly relevant for extended emission, we computed an exposure map for the analysis

3 At 3 arcmin distance from the optical axis, the radius of $50 \%$ encircled energy of the Chandra point-spread function is still less than $0.8^{\prime \prime}$. 


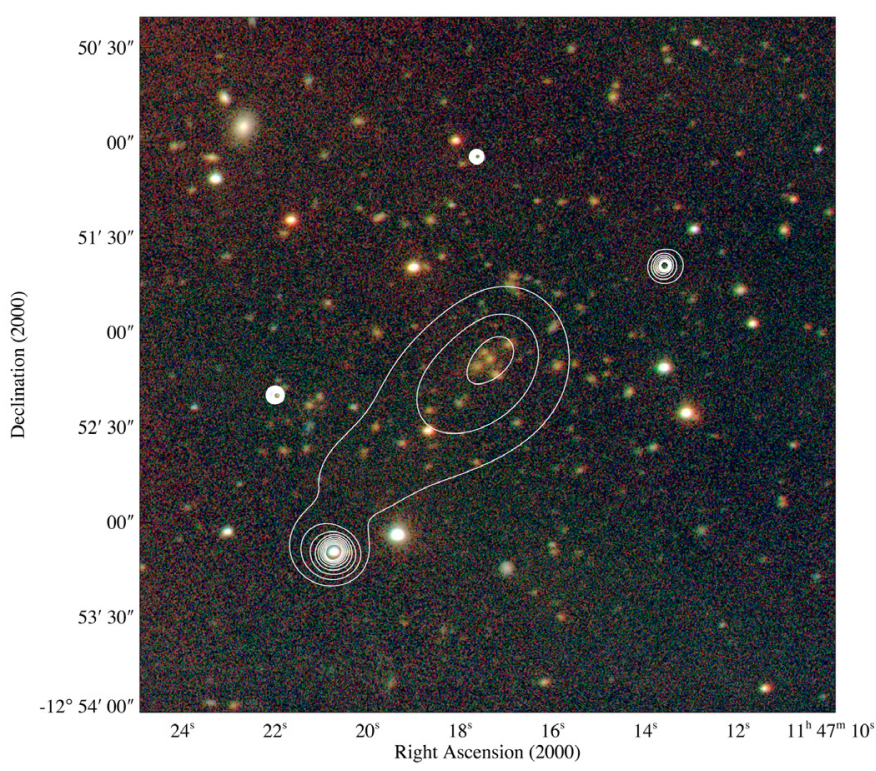

Fig. 4. As Fig. 3, but also showing logarithmically spaced contours of the X-ray surface brightness as observed with Chandra and adaptively smoothed using the ASMOOTH algorithm (Ebeling et al. 2006). Although the bulk of the emission clearly originates from the QSO (point source in the lower left), Chandra also detects diffuse emission from the galaxy cluster. (This figure is available in color in electronic form.)

of the diffuse cluster emission, assuming a monochromatic spectrum of $1.3 \mathrm{keV}$, the energy of the peak of the observed spectrum in the cluster region.

Having divided the X-ray image by the exposure map, we investigate the surface brightness distribution of the extended emission around the cluster position, using various source and background regions the exact definitions of which are refined in an iterative process. A circular source region is centred on the approximate peak of the diffuse emission; background regions are defined by copying the source region to the same CHIPY position on the other three ACIS-I chips. This technique minimizes the impact of any systematic chip-y dependence of the background on the data analysis. The source region is adjusted slightly using the results from our spatial modelling of the observed X-ray surface brightness; specifically, we adopt the location of the peak of our best-fitting two-dimensional model as the centre of the source region, and take the extent of the latter to be the radius at which the signal-to-noise ratio of the onedimensional radial surface-brightness profile falls to $50 \%$ above the background value (see Fig. 7 in Sect. 5.2).

To model the diffuse cluster emission we use the "isothermal King" model which, in projection and for spherically symmetric emission, takes the form of the one-dimensional $\beta$ model (Cavaliere \& Fusco-Femiano 1976)

$f(r)=A\left(1+\left(r / r_{0}\right)^{2}\right)^{-3 \beta+1 / 2}$

where $A$ is the amplitude and $r_{0}$ is the core radius. To describe the spatial distribution of the cluster emission we use the above model (generalised to allow elliptical shapes when applied to the two-dimensional surface brightness) and an additional constant background term. Poisson statistics were used to account for the low photon counts detected in the cluster region.

No spatial fitting was performed for the QSO emission which is pointlike at the resolution of our data; the extraction region used for the spectral analysis was a circle of 8 arcsec radius which captures more than $99.9 \%$ of the QSO flux.

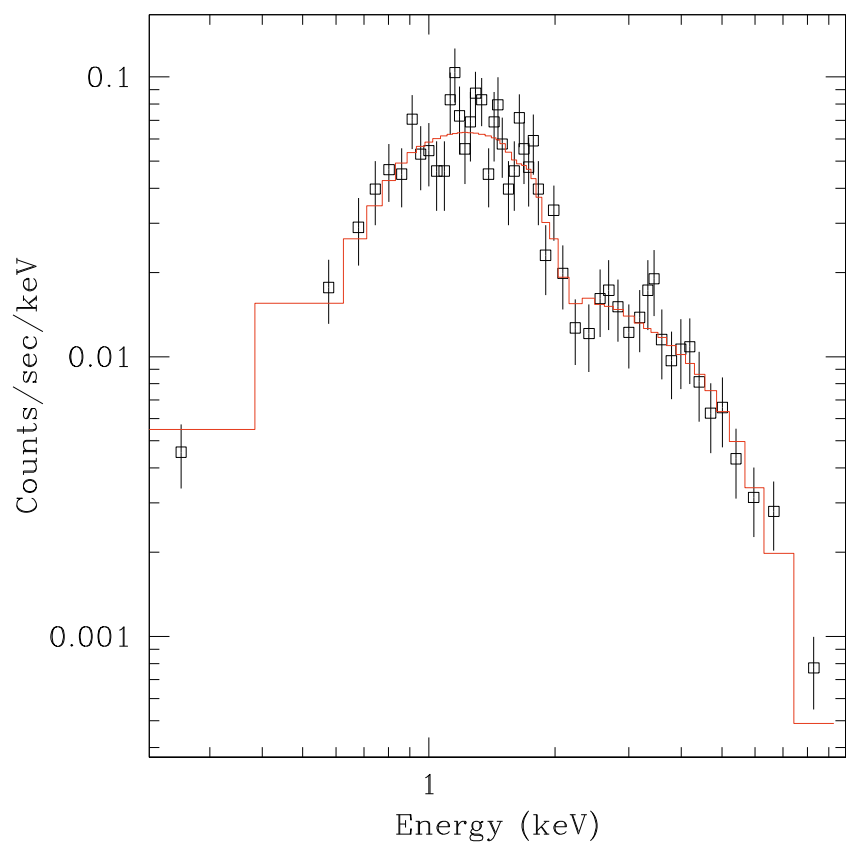

Fig. 5. Spectrum of the QSO g1147207-125310 in the $0.3-8 \mathrm{keV}$ band as obtained with ACIS-I, i.e., folded through the telescope and detector response function. The red line shows the best-fiting power-law model, with $\Gamma=1.4$.

\subsection{Spectral fitting}

We approximate the spectrum of the QSO by a power law, modified by photoelectric absorption within our Galaxy. The absorption model was of the form

$a(E)=\exp \left(-n_{\mathrm{H}} \sigma(E)\right)$

where $\sigma(E)$ is the effective energy-dependent photoelectric absorption cross-section per hydrogen atom (Morrison \& McCammon 1983), and $n_{\mathrm{H}}$ is the equivalent column density of neutral hydrogen in the direction of the QSO. The power law was parameterised as

$f(E)=A E^{-\Gamma}$

where $A$ is the amplitude and $\Gamma$ is the photon index.

For the emission from the cluster we assumed a MeweKaastra-Liedahl thermal plasma model (Mewe et al. 1985; Mewe et al. 1986; Kaastra 1992; Liedahl 1995), modified by the same photoelectric absorption model as used for the QSO (Eq. (2)). For both cluster and QSO emission, we grouped the extracted spectra into bins of 20 photons each before subtracting a suitably scaled background and, finally, spectral fitting with the SHERPA package which is part of the CIAO software suite.

\section{Results}

\subsection{QSO}

A total of 1208 net photons were detected within the QSO source region, enough to constrain the two fit parameters of our model (Eqs. (2), (3)). We find the best-fit value for $n_{\mathrm{H}}$ of $(2.74 \pm$ $1.8) \times 10^{20} \mathrm{~cm}^{-2}$ to be consistent with the Galactic value of $2.92 \times 10^{20} \mathrm{~cm}^{-2}$ (Dickey \& Lockman 1990), and conclude that self-absorption at the source is negligible.

Figure 5 shows the observed QSO spectrum and the bestfitting power-law model in the $0.3-8 \mathrm{keV}$ range with $n_{\mathrm{H}}$ frozen 

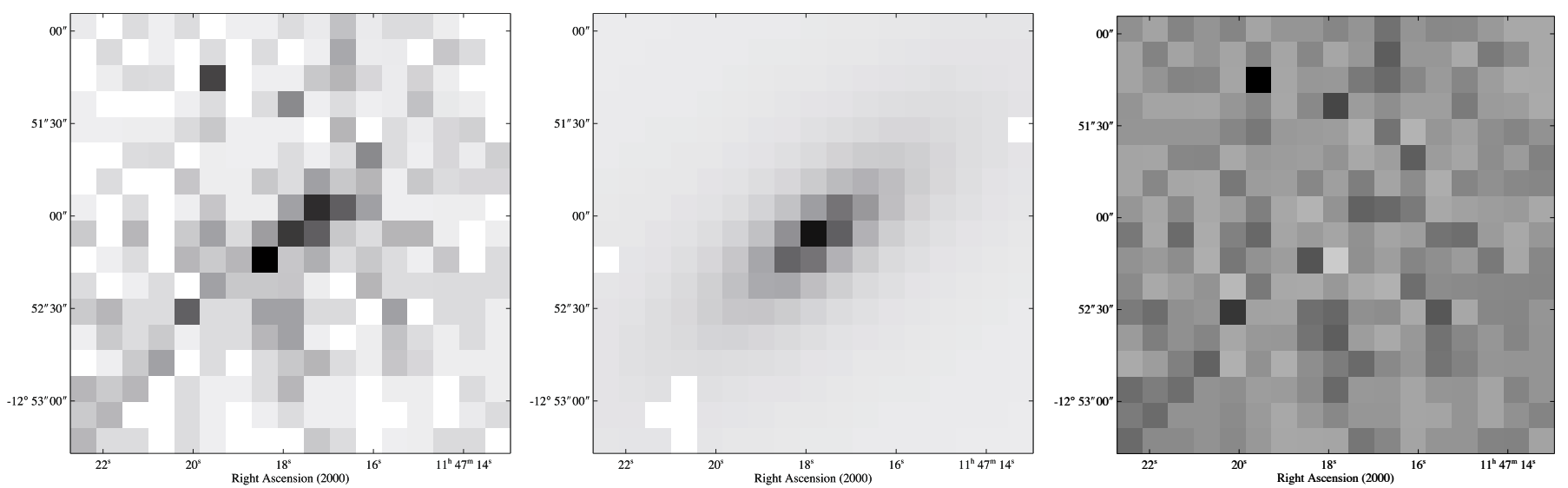

Fig. 6. Left: X-ray-surface-brightness image of the data in the cluster region, after division by the exposure map and binned into $8 \times 8$ arcsec ${ }^{2}$ pixels. Centre: best-fitting two-dimensional $\beta$ model. Right: residuals. The scaling is linear in all three panels.

at the Galactic value. We find the model to provide an adequate description of the data $\left(\chi^{2}\right.$ of 35.4 for 50 degrees of freedom, i.e. a reduced $\chi^{2}$ value of 0.71 ) and measure $\Gamma=1.4 \pm 0.1$ for the photon index.

From the best-fitting model and the known QSO redshift of $z=0.496$ we derive an unabsorbed flux of $(1.4 \pm 0.1) \times$ $10^{-12} \mathrm{erg} \mathrm{s}^{-1} \mathrm{~cm}^{-2}$ and a luminosity in the QSO rest frame of $(8.0 \pm 0.8) \times 10^{44} \mathrm{erg} \mathrm{s}^{-1}$ (both in the $0.3-8 \mathrm{keV}$ band). In the ROSAT $0.1-2.4 \mathrm{keV}$ band, the corresponding values are $(6.8 \pm 0.4) \times 10^{-13} \mathrm{erg} \mathrm{s}^{-1} \mathrm{~cm}^{-2}$ and $(5.1 \pm 0.2) \times 10^{44} \mathrm{erg} \mathrm{s}^{-1}$.

\subsection{Cluster}

A total of 592 photons were detected within 1 arcmin of the peak of the cluster emission, of which 253 can be attributed to the cluster.

As described in Sect. 4 we fit the two-dimensional X-ray surface-brightness distribution of the cluster with an elliptical $\beta$ model plus a constant background. We account for the poor photon statistics by binning the data heavily into $8 \times 8 \operatorname{arcsec}^{2}$ pixels and, in addition, use the Cash statistic, rather than $\chi^{2}$, in the fitting process. The best-fitting model (Fig. 6) is characterised by $\beta=0.43 \pm 0.1$, a core radius of $r_{0}=(87 \pm 38) \mathrm{kpc}$, and a very pronounced (and statistically significant) eccentricity of $0.6 \pm 0.1$. A formal goodness of fit cannot be trivially computed from the Cash statistic; a simple visual inspection of Fig. 6, however, suggests that our simple spatial model does not allow an adequate description of the true photon distribution.

The azimuthally averaged one-dimensional radial profile of the cluster emission, extracted around the centre of the bestfitting two-dimensional model, is shown in Fig. 7. We fit again a $\beta$ model plus constant background (Eq. (1)) and find $\beta=$ $0.54 \pm 0.10$, a core radius of $r_{0}=(145 \pm 52) \mathrm{kpc}$, consistent with the results from the two-dimensional fit. The goodness of fit as given by the $\chi^{2}$ statistic is 1.1 (reduced $\chi^{2}$, i.e. per degree of freedom), much better than the value obtained before for our two-dimensional model.

Modelling the cluster spectrum (Fig. 8) as described in Sect. 4 we find both the metal abundance and the absorption term to be unconstrained by the small number of net photons detected from the cluster. We therefore freeze the metal abundance at a value of 0.3 (abundance by number relative to solar as in Anders \& Grevesse 1989), a value within the typical range of abundances observed in galaxy clusters (Arnaud et al. 1992; Balestra et al. 2007), and set $n_{\mathrm{H}}$ to the Galactic value used

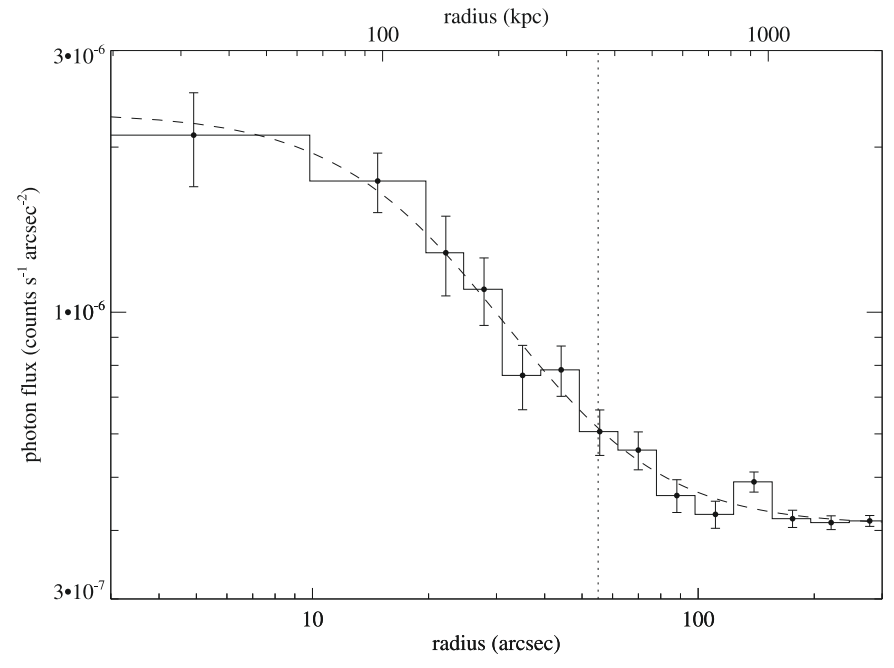

Fig. 7. Radial surface brightness profile of the X-ray emission from the cluster; the best-fitting $\beta$ model is shown by the dashed line. The vertical dotted line marks the radius within which the signal exceeds the constant background by at least $50 \%$ (the source region for our spectral fits).

before. The sole remaining truly free model parameter is then the temperature of the intra-cluster gas for which we measure $k T=(6.7 \pm 4.4) \mathrm{keV}$.

Using this model we derive unabsorbed fluxes of $(2.2 \pm 0.3) \times$ $10^{-13} \mathrm{erg} \mathrm{s}^{-1} \mathrm{~cm}^{-2}$ and $(1.2 \pm 0.2) \times 10^{-13} \mathrm{erg} \mathrm{s}^{-1} \mathrm{~cm}^{-2}$ in the $0.3-8 \mathrm{keV}$ and $0.1-2.4 \mathrm{keV}$ band, respectively. The corresponding intrinsic luminosities at the cluster redshift of $z=0.58$ are then $(2.7 \pm 0.4) \times 10^{44} \mathrm{erg} \mathrm{s}^{-1}$ and $(1.3 \pm 0.2) \times 10^{44} \mathrm{erg} \mathrm{s}^{-1}$ in the same two passbands.

\section{Summary and discussion}

Our Chandra/ACIS-I observation of the RASS source 1RXSJ114720.0-125253 shows the bulk of the X-ray emission $(85 \%)$ to originate from the QSO g1147207-125310 $(z=0.496)$ rather than the galaxy cluster LCDCS S031 $(z=0.58)$. Their combined flux as measured with ACIS-I is lower than the earlier RASS estimate but both measurements agree within the errors. Here we briefly discuss our findings for either object.

The X-ray luminosity of the QSO of $(8.0 \pm 0.8) \times$ $10^{44} \mathrm{erg} \mathrm{s}^{-1}(0.3-8 \mathrm{keV})$ falls easily within the wide range (almost three orders of magnitude) of luminosities measured for 


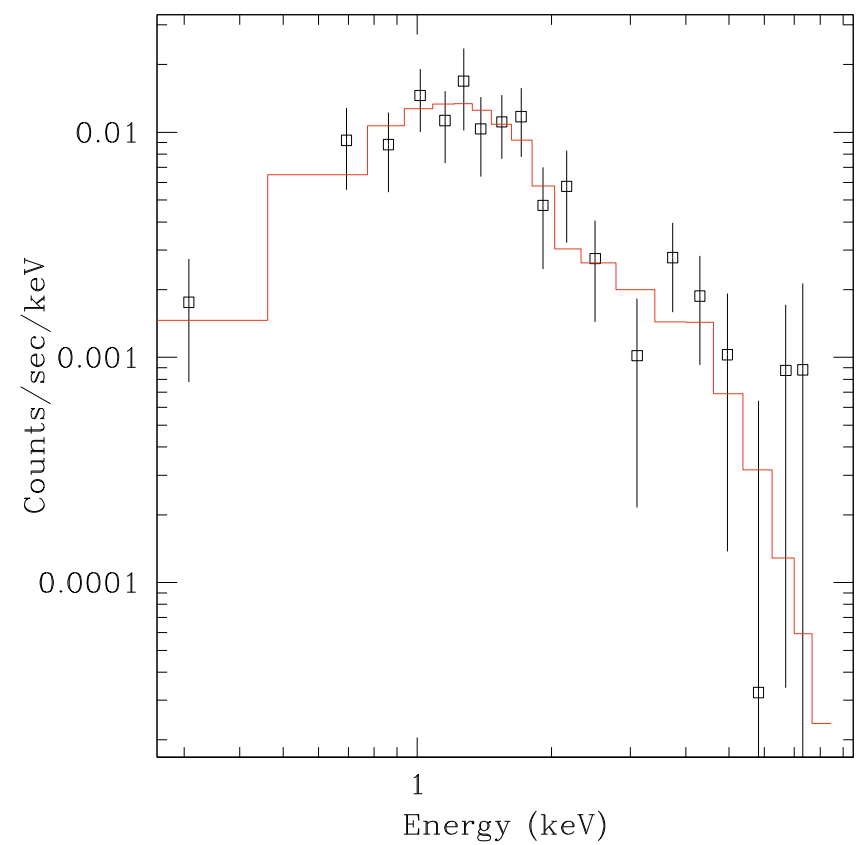

Fig. 8. Spectrum of the cluster LCDCS S031 in the energy range $0.3-8 \mathrm{keV}$. The red line shows the best-fitting XSMEKAL model, characterized by a plasma temperature of $k T=(6.7 \pm 4.4) \mathrm{keV}$.

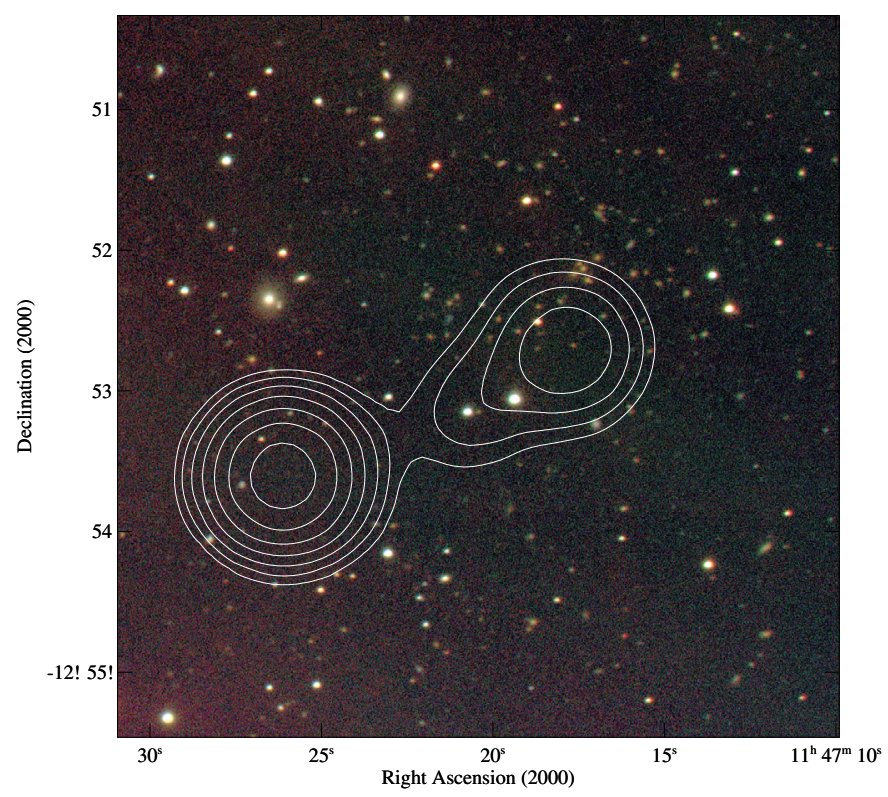

Fig. 9. Shown here are radio emission contours overlaid on an optical image. The QSO lies in between both radio sources. While the source to the right appears as a extended lobe, the other source may be its counterpart, or simply a point source.

ROSAT-detected QSOs (Dewangan et al. 2002). The only existing optical spectrum (Fig. 2) shows a broad MgII line but no other clear features at 4000-7500 ̊ that would allow a quantitative analysis beyond the measurement of the object's redshift. The QSO's X-ray spectrum is well described by a power law with photon index $\Gamma=1.4$, a value that is typical for radio-loud QSOs (e.g. Canizares et al. 1989). Examination of the radio data obtained by the NVSS at $1.4 \mathrm{GHz}$ reveals the presence of two radio sources on either side of the QSO (Fig. 9), one pointlike (and lacking an obvious optical counterpart), the other one more diffuse (and close to LCDCS S031). At distances of more than
$300 \mathrm{kpc}$ from the QSO, it is not obvious though that either of these sources is physically associated with g1147207-125310. The western, diffuse radio source could conceivably be a radio relic at the periphery of LCDCS S031 which would suggest a recent merger event (e.g., Hoeft \& Brueggen 2007). Deeper, highresolution radio observations, preferably at multiple frequencies, would be required to better determine the origin of the radio emission.

Our analysis of the X-ray emission from the distant galaxy cluster LCDCS S031 is hampered by the poor photon statistics from this short Chandra observation, but yields an X-ray luminosity of $(2.7 \pm 0.4) \times 10^{44} \mathrm{erg} \mathrm{s}^{-1}(0.3-8 \mathrm{keV})$ and a crude estimate of the temperature of the intra-cluster gas of $(6.7 \pm 4.4) \mathrm{keV}$, consistent with the value of $3.8 \mathrm{keV}$ expected from the luminosity-temperature relation for galaxy clusters (White et al. 1997). This makes LCDCS S031 a typical representative of the population of clusters at $z=0.5-0.7$ as probed by serendipitous ROSAT X-ray cluster surveys such as WARPS or the 400 sq. deg. survey (Perlman et al. 2002; Horner et al. 2008; Burenin et al. 2007), as opposed to the extremely X-ray luminous systems compiled by MACS (Fig. 1). Our spatial description of the X-ray emission from LCDCS S031 based on simple $\beta$ models yields estimates of about $100 \mathrm{kpc}$ for the core radius and a $\beta$ value of 0.5 , values that both lie at the lower end of the range typically observed for clusters (e.g. Jones \& Forman 1984). We note, however, the marginal agreement between the best-fit values obtained from two- and one-dimensional spatial fits, as well as the significant eccentricity of 0.6 found in our fits, all of which suggest an unrelaxed, disturbed morphology.

\section{The likelihood of QSO/cluster alignments in shallow X-ray surveys}

The poor photon statistics and limited angular resolution of the RASS data make the secure identification of galaxy clusters from X-ray source properties alone unfeasible for all but the brightest and most nearby systems. Optical follow-up thus plays a crucial role in RASS-based cluster surveys ${ }^{4}$ like MACS, which - in particular for increasingly faint X-ray sources - leads to the problem of multiple plausible optical counterparts competing for the ID. We here discuss the specific case of QSOs in (or superimposed onto) galaxy clusters and attempt to determine the most plausible ID.

\section{1. $1 R X S J 114720.0-125253$}

As we shall show, the case of 1RXSJ114720.0-125253 is rare, but useful anyway to introduce and illustrate a simple probabilistic argument that can be applied to any potential QSO/cluster alignment. Instead of trying to answer the most general (and non-trivial) question of which fraction of the observed X-ray flux most probably originates from which source (QSO or cluster) we limit ourselves to the question of which source is more likely to dominate the X-ray emission and adopt a value of $90 \%$ to quantify the required fraction of the flux. In the absence of supporting evidence from, e.g., the shape of the X-ray spectrum or the X-ray source extent, the only quantities that can be used to assign relative probabilities are the (comoving) space density of either type of object and the search volume, which is straightforward to estimate if the redshifts of the cluster and the QSO

\footnotetext{
${ }^{4}$ Although we here focus on the RASS, the same arguments apply to cluster searches near the flux limit of other low-resolution X-ray surveys, e.g., eROSITA.
} 
are known. Note that, in the following, we adopt $h_{0}=0.5$ and an Einstein-de Sitter cosmology since this is the cosmology assumed in the relevant literature for the computation of the X-ray luminosity functions of QSOs and clusters.

Starting with the hypothesis that the cluster LCDCS S031 $(z=0.58)$ is responsible for the bulk of the X-ray emission observed in the RASS, we use the cluster X-ray luminosity function at $z \sim 0.6$ from Mullis et al. (2004) to estimate the space density of such systems and find a value of about $5 \times 10^{-10} \mathrm{Mpc}^{-3}$. The search volume (assumed to be a shell of width $\delta z=0.1$ around the cluster redshift) is $1.8 \times 10^{9} \mathrm{Mpc}^{3} \mathrm{sr}^{-1}$, leading to an expectation value of roughly $1 \mathrm{sr}^{-1}$. In this scenario, the QSO g1147207-125310 would be a serendipitous object with a minority contribution of only $10 \%$ of the X-ray flux. The space density of such QSOs can be estimated from the X-ray luminosity functions determined by Miyaji et al. (2000) and is given by about $2 \times 10^{-6} \mathrm{Mpc}^{-3}$. Multiplication with the relevant comoving volume (a shell of width $\delta z=0.1$ around the QSO redshift) yields an expectation value of $6600 \mathrm{sr}^{-1}$. For the inverse case where most of the RASS flux originates from g1147207-125310 at $z=0.496$ and only $10 \%$ from the chance alignment with LCDCS S031 at $z=0.58$, the corresponding expectation values are 663 QSOs $\mathrm{sr}^{-1}$ and 725 clusters $\mathrm{sr}^{-1}$. Hence, the ratio of the conditional probabilities for these two scenarios (LCDCS S031 dominates and g1147207-125310 is a low-luminosity serendipitous source, and vice versa) is given by $(1 / 426) \times(3300 / 725)=0.014$, i.e. the probability of the cluster emission dominating is $1.4 \%$.

Applying these expectation values to the solid angle covered by MACS and taking into account the number of RASS sources screened during the identification phase of the project, we arrive at the prediction that only one chance alignment akin to 1RXSJ114720.0-125253 within the RASS error circle is in fact expected within the MACS survey area - in excellent agreement with our observation.

\section{2. $H 1821+643$}

The same argument as above can be applied to the interesting case of H1821+643, an X-ray bright QSO within the rich galaxy cluster CL $1821+643(z=0.299$, Schneider et al. 1992; Hall et al. 1997; Russell et al. 2009).

We first note that, in the case of H1821+643, the fact that the QSO is a cluster member does not affect our probabilistic argument, unless the X-ray emission from the QSO is in fact triggered or boosted by its cluster environment (which is not entirely clear for H1821+643, Russell et al. 2009). When addressing the question of which component is more likely to dominate the X-ray emission, based on RASS data alone, we can also ignore any search-volume considerations since cluster and QSO are at the same redshift. The RASS flux detected from this source in the $0.1-2.4 \mathrm{keV}$ band is $2.0 \times 10^{-11} \mathrm{erg} \mathrm{s}^{-1} \mathrm{~cm}^{-2}$, making it almost 20 times as X-ray bright as 1RXSJ114720.0-125253. If the emission originated solely from the cluster, the X-ray luminosity of the latter would be $4.7 \times 10^{45} \mathrm{erg} \mathrm{s}^{-1}\left(h_{0}=0.7, \Lambda \mathrm{CDM}\right)$, making it the most X-ray luminous cluster known (Fig. 1). It is thus immediately clear that the QSO is likely to dominate. A chance alignment is unlikely though even for the 90:10 flux ratio between the dominant and the serendipitous source adopted before, as the frequency of clusters featuring $L_{X}=5 \times 10^{44} \mathrm{erg} \mathrm{s}^{-1}$ at $z \sim 0.3$ is only $9 \mathrm{sr}^{-1}$.

In-depth follow-up was thus clearly warranted, and indeed rewarded by a measurement of $1.5 \times 10^{45} \mathrm{erg} \mathrm{s}^{-1}\left(h_{0}=0.7\right.$, $\Lambda \mathrm{CDM}$ ) for the X-ray luminosity of CL $1821+643$, excluding the central cool-core component (Hall et al. 1997), which makes the system one of the 20 most X-ray luminous clusters known within $z=0.3$.

Acknowledgements. We thank C.J. Ma for advice on many aspects of the reduction of Chandra data, and an anonymous referee for the suggestion to include a discussion of the probability of identification conflicts caused by clusterQSO alignments. Financial support from SAO grant GO9-0137X is gratefully acknowledged.

\section{References}

Allen, S. W., Schmidt, R. W., Fabian, A. C., \& Ebeling, H. 2003, MNRAS, 342, 287

Anders, E., \& Grevesse, N. 1989, Geochim. Cosmochim. Acta, 53, 197

Arnaud, M., Rothenflug, R., Boulade, O., Vigroux, L., \& Vangioni-Flam, E. 1992, A\&AS, 254, 49

Balestra, I., Tozzi, P., Ettori, S., et al. 2007, A\&A, 462, 429

Borgani, S., Rosati, P., Tozzi, P., et al. 2001, ApJ, 561, 13

Burenin, R. A., Vikhlinin, A., Hornstrup, A., et al. 2007, ApJS, 172, 561

Cavaliere, A., \& Fusco-Femiano, R. 1976, A\&A, 49, 137

Canizares, C. R., \& White, J. L. 1989, ApJ, 339, 27

Dewangan, G. C., Singh, K. P., Gunn, K. F., et al. 2002, MNRAS, 337, 693

Dickey, J., \& Lockman, F. 1990, ARAA, 28, 215

Ebeling, H., Edge, A. C., \& Henry, J. P. 2001, ApJ, 553, 668

Ebeling, H., White, D. A., \& Rangarajan, F. V. N. 2006, MNRAS, 368, 65

Ebeling, H., Barrett, E., Donovan, D., et al. 2007, ApJ, 661, L33

Ebeling, H., Edge, A. C., Mantz, A., et al. 2010, MNRAS, 407, 83

Gonzales, A., Zaritsky, D., Dalcanton, J., \& Nelson, A. 2001, ApJS, 137, 117

Hall, P. B., Ellingson, E., \& Green, R. F. 1997, AJ, 113, 1179

Henry, J. P. 2000, ApJ, 534, 565

Hicks, A. K. 2008, ApJ, 680, 1022

Hoeft, M., \& Brueggan, M. 2007, 375, 77

Horesh, A., Maoz, D., Ebeling, H., Seidel, G., \& Bartelmann, M. 2010, MNRAS, 406, 1318

Horner, D. J., Perlman, E. S., Ebeling, H., et al. 2008, ApJS, 176, 374

Jones, C., \& Forman, W. 1984, ApJ, 276, 38

Jones, D. H., Read, M. A., Saunders, W., et al. 2009, MNRAS, 399, 683

Kaastra, J. S. 1992, An X-Ray Spectral Code for Optically Thin Plasmas (Internal SRON-Leiden Report, updated version 2.0)

Liedahl, D. A., Osterheld, A. L., \& Goldstein, W. H. 1995, ApJ, 438, L115

Mantz, A., Allen, S. W., Ebeling, H., \& Rapetti, D. 2008, MNRAS, 397, 1179

Mewe, R., Gronenschild, E. H. B. M., \& van den Oord, G. H. J. 1985, A\&AS, 62, 197

Mewe, R., Lemen, J. R., \& van den Oord, G. H. J. 1986, A\&AS, 65, 511

Miyaji, T., Hasinger, G., \& Schmidt, M. 2000, A\&A, 353, 25

Morrison, R., \& McCammon, D. 1983, ApJ, 270, 119

Mullis, C. R., Vikhlinin, A., Henry, J. P., et al. 2004, ApJ, 607, 175

Perlman, E. S., Horner, D. J., Jones, L. R., et al. 2002, ApJS, 140, 265

Pierpaoli, E., Borgani, S., Scott, D., \& White, M. 2003, MNRAS, 342, 163

Voges, W., Aschenbach, B., Boller, Th., et al. 1999, A\&A, 349, 389

White, D. A., Jones, C., \& Forman, W. 1997, MNRAS, 292, 419 